\title{
Oesophageal subepithelial fibrosis: an extension of oral submucosal fibrosis
}

\author{
S P Misra, Vatsala Misra, Manisha Dwivedi, S C Gupta
}

\begin{abstract}
Summary
Fifty-five patients with oral submucosal fibrosis and an equal number of patients with no evidence of the disease were studied. All patients underwent upper gastrointestinal endoscopy and any abnormality was noted. Multiple oesophageal biopsies were obtained from the upper end of the oesophagus and from any endoscopically observed abnormality. The histological changes in the two groups were assessed blindly by an experienced histopathologist. Histological abnormalities were noted in the oesophageal mucosa in $2 \%$ of controls and $66 \%$ of patients with oral submucosal fibrosis $(p<0.0001)$. In the control group, acanthosis was seen in one patient, while in the patient group atrophy of the squamous epithelium was evident in $52 \%$, hyperkeratosis in $52 \%$, parakeratosis in $30 \%$, dyskeratosis in $14 \%$, acanthosis in $14 \%$, and papillomatosis and mild dysplasia in $2 \%$ patients. Subepithelial collagenization was seen in $32(64 \%)$ patients. The oesophageal abnormalities were seen more frequently in patients who had consumed Pan masala, Gutka, betel nut, tobacco or a combination of some or all of these, with or without betel leaf, for $\geqslant 5$ years compared to those consuming them for a shorter period of time ( $91 \%$ vs $46 \%$, $p<0.001$ ). It is concluded that oral submucosal fibrosis is not a disease confined to the oral cavity; the oesophagus may also be involved in about two-thirds of patients.
\end{abstract}

Keywords: oral submucosal fibrosis; oesophageal subepithelial fibrosis; betel; Indians; chewing tobacco

Oral submucosal fibrosis (OSMF) is a chronic irreversible disease of unknown aetiology. It is mainly found in Indians ${ }^{12}$ and affects about $0.2-1.2 \%$ of the urban population attending dental clinics in India. ${ }^{3-5}$ It has also been seen among Indians living in Kenya, Malaysia, Uganda, South Africa, Fiji and the UK, ${ }^{6-10}$ and cases have been reported from ethnic groups in Taiwan, Nepal, Thailand, Vietnam and Sri Lanka. ${ }^{11-13}$ The disease leads to fibro-elastic transformation of the lamina propria and epithelial atrophy of the oral mucosa. Later, the oral mucous membrane becomes stiff, leading to trismus. Although the aetiology is not known, it has been postulated that the disease is caused by irritation of the oral submucosa by irritants such as capsaicin in chillies and tannins in betel nuts. ${ }^{10}{ }^{14}$ Genetic and environmental factors have also been blamed for the disease. ${ }^{15}$ As the name denotes, the disease mostly affects the oral mucosa and, although involvement of the oesophageal mucosa has occasionally been reported, ${ }^{1}$ there is a paucity of reports describing oesophageal involvement. This study was set up to study the prevalence of oesophageal disease in patients with OSMF.

\section{Materials and methods}

The study group consisted of 55 patients with OSMF and 55 other patients with no evidence of OSMF who were undergoing upper gastrointestinal endoscopy. Patients with oesophageal disease (eg, gastro-oesophageal reflux disease, carcinoma of the oesophagus, oesophageal stricture or oesophageal varices) were excluded. The diagnosis of OSMF was made on clinical grounds and buccal biopsy in 36 patients and on clinical grounds alone in the remaining 19 patients. OSMF was clinically classified as severe if the patient could not open his mouth to accommodate a paediatric mouthguard with an outer diameter of $20 \mathrm{~mm}$, during upper gastrointestinal endoscopy.

A detailed clinical history was obtained, including the duration of the disease and symptoms, and information on any substance(s) chewed and the duration of consumption. Thereafter, all subjects underwent upper gastrointestinal endoscopy with a forward-viewing endoscope (Olympus model XQ 20 or XP 20, Olympus Optical Corporation, Tokyo). A specially designed mouthguard was used in patients who could not open their mouth fully for even a paediatric mouthguard to be inserted. ${ }^{16} \mathrm{~A}$ careful search was made for any abnormality during endoscopy, especially in the oesophagus. Multiple oesophageal biopsies were obtained from any abnormality noticed during endoscopy, and in all cases from the upper end of the oesophagus, just below the upper oesophageal sphincter. All biopsies were fixed in $10 \%$ formalin and sent for histopathological examination.

After routine processing, 3-5 $\mu \mathrm{m}$ sections were cut from paraffin wax embedded sections, stained with haematoxylin and eosin, and evaluated by a histopathologist who was unaware of the clinical diagnosis or endoscopic findings. The sections were examined for histological changes. In the epithelium, evidence of atrophy of the lining squamous epithelium, hyperkeratosis, parakeratosis, 
dyskeratosis, acanthosis, papillomatosis and dysplasia was sought. In the subepithelial tissue, evidence of collagenization was sought, which was subjectively graded as mild or severe by the histopathologist. Oesophageal biopsies which were superficial and did not contain subepithelial tissue were not evaluated.

\section{STATISTICS}

The two groups were compared by the Student's $t$-test, $\chi^{2}$-test with or without Yates' correction and the Fischer's exact test.

\section{Results}

CONTROLS

The mean age of the controls was $31.2 \pm 11.8$ years; $45 / 55$ were males. None of these subjects showed any oesophageal abnormality on endoscopy. Oesophageal biopsies were too superficial for proper interpretation in six subjects and were discarded. Of the other 49 subjects, oesophageal biopsy from one subject showed evidence of acanthosis in the epithelial tissue. There was, however, no evidence of collagenization of the lamina propria. No histologic abnormality was seen in the oesophageal biopsies from the other 48 subjects (figure 1 ).

\section{PATIENTS}

The mean age of this group was $29.4 \pm 10.6$ years; $47 / 55$ were males. The age and sex ratio were not significantly different from the control group. One patient was asymptomatic and was found to have OSMF accidentally when he could not open his mouth fully during endoscopic examination to detect the cause of melaena. All patients except one chewed either Pan masala, Gutka, betel nut, tobacco or a combination of some or all of these, with or without betel leaf. All 54 of these patients consumed betel nut in one form or another. Only two patients smoked cigarettes. The offending substance had been chewed for a mean \pm SD of $6.2 \pm 6.4$ years (range 0.5-25). One 25-yearold man with a history of inability to open his mouth fully for the last year denied the use of any offending agent. This was corroborated by his parents and siblings. The mean \pm SD duration of symptoms in the patients was $18 \pm 32.8$ months (range $0.5-168$ ).

All patients had difficulty in opening their mouths. It was clinically severe in $23(42 \%)$ patients in whom a specially designed mouthguard had to be used for upper gastrointestinal endoscopy. Thirty-nine (71\%) complained of burning sensations in the mouth while eating highly seasoned food and $21(38 \%)$ patients had ulcers in their mouth. Oesophageal symptoms were noted in seven (13\%) patients. Dysphagia was reported by four $(7 \%)$ patients and odynophagia by two patients. One patient had both dysphagia and odynophagia. Loss of taste sensation was noticed by two patients and occasional bleeding from the cheek by one patient.

None of the patients showed any abnormality during upper gastrointestinal endoscopy. Oesophageal biopsies were too superficial for proper interpretation in five $(9 \%)$ patients. Of

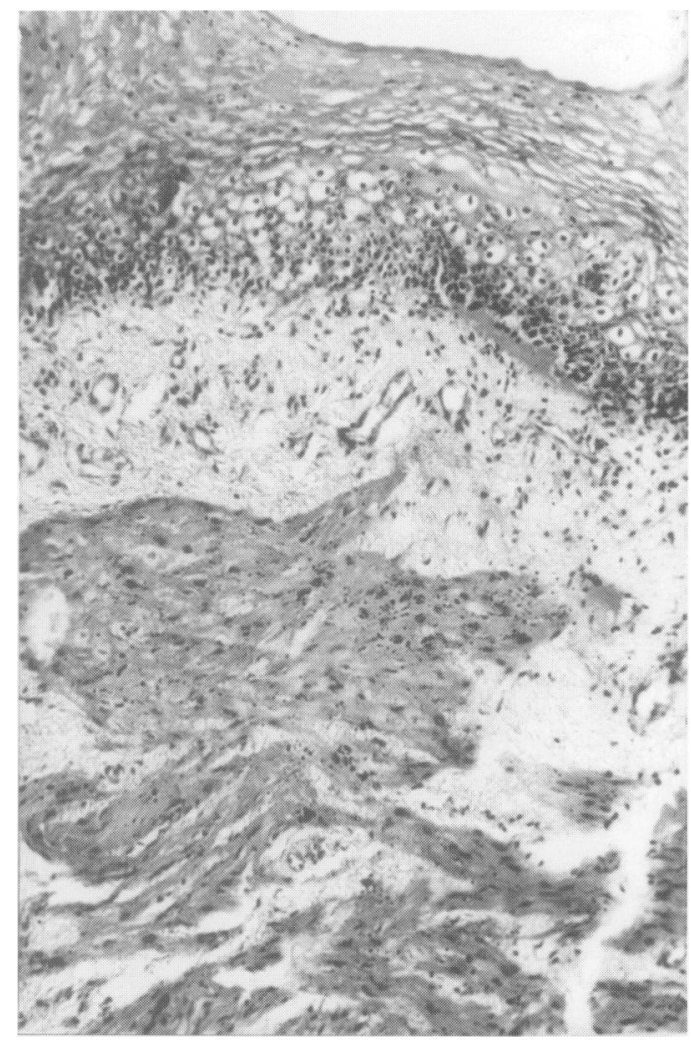

Figure 1 Oesophageal biopsy from a control subject. Note the normal lamina propria $(\mathrm{H} \& \mathrm{E}$, orig $\times 80)$

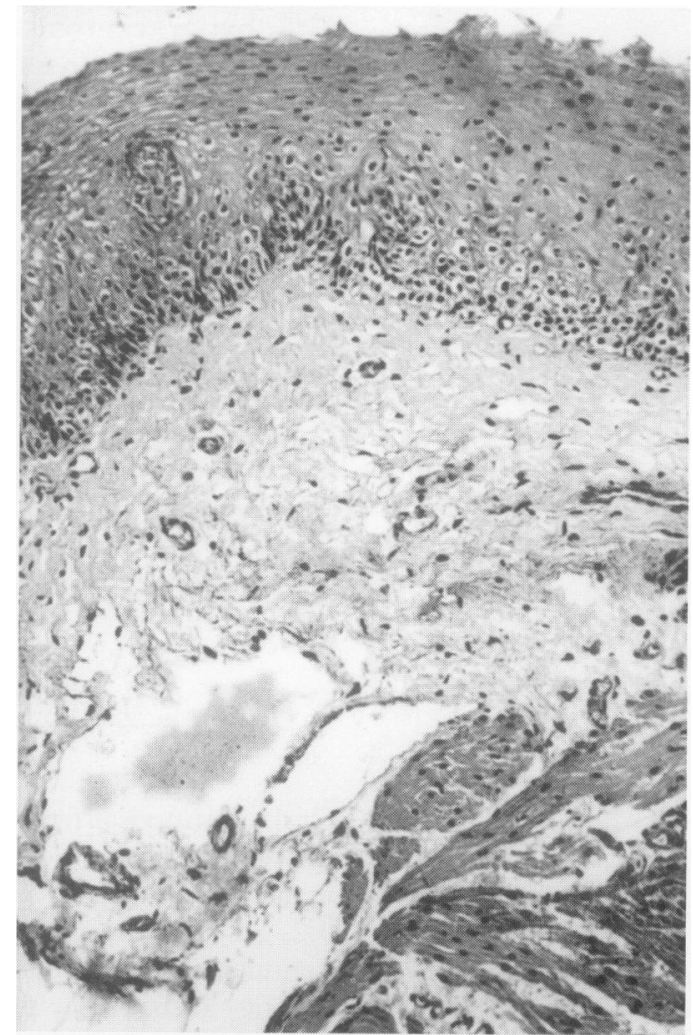

Figure 2 Oesophageal biopsy from a patient with oral submucosal fibrosis showing mild collagenization of the lamina propria ( $\mathrm{H} \& \mathrm{E}$ orig $\times 80)$

the 50 remaining patients, histological abnormalities were noted in $33(66 \%)$. The difference from the control group was statistically highly significant $(p<0.0001)$. Atrophy of the 


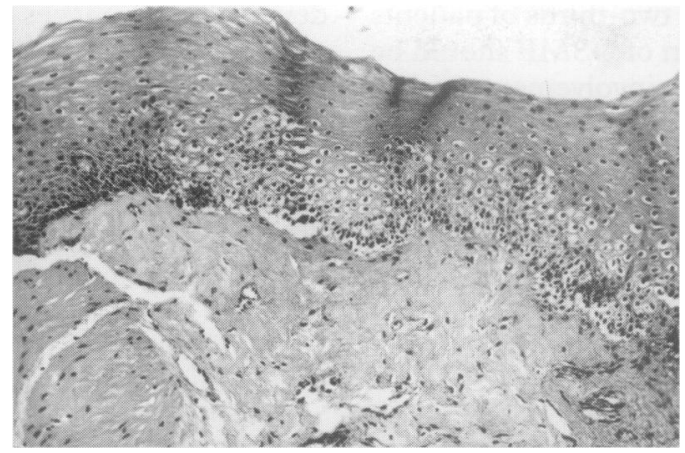

Figure 3 Severe collagenization of the lamina propria in a patient with oral submucosal fibrosis (H\&E orig $\times 80$ )

squamous epithelium was evident in $26(52 \%)$, hyperkeratosis in $26(52 \%)$, parakeratosis in 15 $(30 \%)$, dyskeratosis in $7(14 \%)$ and acanthosis in seven (14\%) patients. Papillomatosis and mild dysplasia were evident in one $(2 \%)$ patient each. Subepithelial collagenization was seen in $32(64 \%)$ patients; it was mild in $15(30 \%)$ (figure 2 ) and severe in 17 (34\%) patients (figure 3).

Histological abnormalities in the oesophageal biopsies were seen in 13 of $28(46 \%)$ patients with a history of consuming one or more of the offending agents listed above for < 5 years compared to 20 of $22(91 \%)$ consuming them for $\geqslant 5$ years. The difference between the two groups was statistically highly significant ( $p<0.001$ ). Histological abnormalities were seen in 14 of $20(70 \%)$ patients with clinically severe OSMF compared to 19 of 30 (63\%) patients with clinically milder disease. The difference between these two groups was not statistically significant. Of the seven patients with oesophageal symptoms, six had histologically severe collagenization (figure 3), and five had been consuming the offending agent(s) for $\geqslant 5$ years. The other two had consumed the offending agents for 2 and 3 years, respectively. The patient with idiopathic OSMF did not have any histologic abnormality in his oesophageal biopsy.

\section{Discussion}

Oral submucosal fibrosis is not an uncommon condition in Indian subjects consuming betel nut, tobacco, Pan masala and Gutka, with or without betel leaf. The disease is also common in expatriates from the Indian subcontinent in the developed countries, especially in the UK where it has been reported from London and other cities. ${ }^{6-10}$ It has been estimated that in the city of Leicester one may find as many as $\mathbf{3 0 0 0}$ cases of OSMF. In a study from Durban, with a large $(46 \%)$ Indian population, a prevalence of $3.4 \%$ has been reported. ${ }^{17}$ The importance of the disease lies in the fact that it is a preventable precancerous condition of the oral cavity which leads to substantial morbidity especially in the younger population.

While OSMF is predominantly a disease of the oral cavity, fibrosis is also known to extend into the pharynx via the pillars and down to the pyriform fossa. It appears logical that the oesophagus should also be involved because at least some of the material which is chewed or kept in the mouth will go down the oesophagus, leading to irritation of the oesophageal mucosa, which is similar to that of the oral cavity. However, although involvement of the oesophagus has been reported, ${ }^{1}$ there are no confirmatory reports to substantiate this. A MEDLINE search revealed only one article on the subject. ${ }^{18}$ In this study by Maher et al, only the endoscopic appearance of the oesophageal mucosa was evaluated because the oesophageal biopsies obtained were too superficial in nature to be of any value. The authors noted that the mucosa was whitish pale in two of the 30 patients studied, stiff and fibrotic in 19, leathery in four, and firm or gritty in another two cases.

In the present study, we were unable to find any endoscopic abnormality in our OSMF patients. However, histologically, oesophageal involvement could be demonstrated in about two-thirds of patients with OSMF. In the majority of cases the disease in the oesophagus was clinically not apparent and oesophageal symptoms were seen in only seven of the 50 (14\%) patients. Of interest was the finding that six out of these seven patients had severe collagenization of the lamina propria of the oesophagus.

Oesophageal subepithelial fibrosis was more common in patients who had consumed betel nut, tobacco, Pan masala or Gutka, with or without betel leaf, for a longer period. However, $46 \%$ of patients had developed the disease after consuming the offending agent(s) for less than 5 years. It therefore appears that, although the chances of developing oesophageal subepithelial fibrosis are high if one of the offending agents are chewed on a long-term basis, even a period of usage as short as a year may be sufficient to cause this condition. This was also evident in a small group of seven patients who had symptoms of dysphagia and odynophagia along with oesophageal subepithelial fibrosis.

One of the patients with OSMF denied consuming any of the known offending agents, which was confirmed by his parents and siblings. There was however, no oesophageal involvement in this patient. Such idiopathic cases of OSMF are known to occur rarely, but the majority of cases occur in people who consume the above-mentioned offending agents.

The implications of oesophageal involvement in patients with OSMF may have far-reaching implications. It is possible that, in at least some of these patients, the lesion will develop into frank carcinoma of the upper oesophagus, as it is well known that OSMF is a premalignant disease. ${ }^{19}$

Although oesophageal subepithelial fibrosis has not been reported earlier in patients with OSMF, ultrastructural abnormalities such as discontinuous, fragmented basement membrane, with reduction of hemidesmosomes, and widened intracellular spaces, have been noted in the oesophageal mucosa of chronic tobacco chewers. ${ }^{20}$ With the changes seen on 
light microscopy in about two-thirds of patients with OSMF, the definition of OSMF should be modified to include the involvement of the oesophagus.

While banning tobacco, betel nut, betel, Panmasala and Gutka is the key to prevention of the disease, this may not be possible in the

1 Jayanthi V, Probert CSJ, Sher KS, Mayberry JF. Oral Jayanthi V, Probert CSJ, Sher KS, Mayberry JF. Oral 6.

Pathak AG. Oral submucous fibrosis. Natl Med $\mathcal{F}$ India 1993;3:249-50

3 Pindborg J, Mehta FS, Gupta PC, Dattary DK. Prevalence of oral submucous fibrosis among 50915 Indian villagers. $B$ $\mathcal{f}$ Cancer 1968;22:646-54.

4 Pindborg JJ, Kalapessi HK, Kale S, Singh B, Talyerkhan $\mathrm{BN}$. Frequency of oral leukoplakia and related condition among 10,000 Bombayites: preliminary report. $\mathcal{F}$ All India Dent Assoc 1965;37:228-9.

5 Zachariah J, Mathew B, Varma NAR, Iqbal AM, Pindborg J. Frequency of oral mucosal lesions among 5000 individuals in Trivandrum, South India. 7 All India Dental Assoc 1966;38:290-6.

6 Kennedy TF, MacDonald DG. Oral submucous fibrosis. A case report. Br Dent f 1968;124:121-4.

7 Moos KF, Madan DK. Submucous fibrosis. Br Dent 7 1968; 125:313-7.

8 McGurk M, Craig GT. Oral submucous fibrosis: two cases of malignant transformation in Asian immigrants to the United Kingdom. Br $\mathcal{F}$ Oral Max Surg 1984;22:56-64.

9 Foster BA. Oral submucous fibrosis: a cause for concern. $B$ Dent $\mathcal{F}$ 1986;160:378.

0 Caniff JP, Harvey W, Harris M. Oral submucous fibrosis: its pathogenesis and management. Br Dent $\mathcal{F} 1986 ; 160: 429-34$ 11 Su JP. Idiopathic scleroderma of the mouth. Report of three cases. Arch Otolaryngol 1954;59:331-2. developing countries because of the lack of political will. However, in the UK, where a large number of immigrants from the Indian subcontinent reside, a blanket ban on import of such articles would go a long way in preventing this precancerous but potentially preventable disease.

12 Pindborg JJ, Sirsat MS. Oral submucous fibrosis. Oral Surg 1966;22:764-79.

13 Shiaw YY, Kwan HW. Submucous fibrosis in Taiwan. Oral Surg 1979;47:453-7

14 Seedat HA, Van Wyk CW. Betel nut chewing and dietary habits of chewers with and without submucous fibrosis and with concomitant oral cancer. S Afr Med f 1988;74:572-5.

15 Lemmer J, Shear M. Oral submucosal fibrosis: A possible case in a person of Caucasian descent. Br Dent Surg 1967;122:343-6.

16 Misra SP, Dwivedi M. A mouthguard for endoscopic examination of patients with oral submucus fibrosis and ankylosis of the temporomandibular joint. Endoscopy 1998;30:S37.

17 Seedat HA, Van Wyk CW. Betel nut chewing and submucous fibrosis in Durban. S Afr Med $\mathcal{F}$ 1988;74:56871 .

18 Maher R, Ahmed W, Qureshi H, Zuberi SJ, Syed S. Oesophageal changes in oral submucous fibrosis using fibreoptic endoscopy - a pilot study. $\mathcal{F}$ Pakistan Med Assoc 1991;41:312-3.

19 Pindborg JJ, Murthy PR, Bhonsle RB, Gupta PC, Daftary DK, Mehta FS. Oral submucous fibrosis as a precancerous condition. Scand F Dent Res 1984;92:224-9.

20 Shankaran K, Kandarkar SV, Contractor QQ, Kalro RH, Desai HG. Ultrastructural changes in oesophageal mucosa of chronic tobacco chewers. Indian $\mathcal{F}$ Med Res 1993; 98:159. 